\title{
Síntese sol-gel de scaffolds porosos de vidro bioativo com adição de agente porogênico
}

\author{
(Sol-gel synthesis of bioactive glass porous scaffolds \\ with addition of porogen agent)
}

\author{
F. B. A. P. Guimarães, B. R. Barrioni, A. C. X. Oliveira, A. A. R. Oliveira, M. M. Pereira \\ Depto. Engenharia Metalúrgica e de Materiais, Escola de Engenharia, Universidade Federal de Minas Gerais, \\ Belo Horizonte, $M G$, Brasil \\ fabianabapg@gmail.com
}

\begin{abstract}
Resumo
O uso de biomateriais capazes de gerar uma resposta biológica tem sido um dos maiores progressos em medicina regenerativa, devido à sua habilidade de suportar o crescimento e estimular a regeneração do tecido danificado. Neste contexto, biocerâmicas, particularmente o vidro bioativo (VB), foram o objetivo de múltiplos estudos. A técnica de adição de agentes porogênicos para a síntese de scaffolds é uma técnica interessante, pois vários tipos de agentes porogênicos podem ser usados. Este estudo propôs obter scaffolds através de quatro agentes porogênicos e avaliar os efeitos que a mudança na temperatura de tratamento poderiam ter em sua cristalinidade. Scaffolds de vidro bioativo obtidos pela técnica sol-gel 100S $\left(100 \% \mathrm{SiO}_{2}\right)$ mais agentes porogênicos (parafina 1, parafina 2, cera e CMC - carboximetilcelulose) foram preparados e caracterizados. Como os melhores resultados foram obtidos com a parafina 1 , scaffolds com composição $58 \mathrm{~S}\left(60 \% \mathrm{SiO}_{2}-36 \% \mathrm{CaO}-4 \% \mathrm{P}_{2} \mathrm{O}_{5}\right)$ e $100 \mathrm{~S}$ usando parafina 1 como agente porogênico foram preparados. Os scaffolds foram submetidos a diferentes temperaturas de tratamento para avaliar a cristalinidade do material. A estrutura de poros foi analisada por microscopia eletrônica de varredura e microtomografia computadorizada e apresentou tamanho, distribuição e porosidade satisfatória, características importantes pois permitem migração celular, transporte de nutrientes, vascularização e crescimento tecidual. Por meio da difração de raios X constatou-se a natureza amorfa dos scaffolds. A $900{ }^{\circ} \mathrm{C}$, scaffolds de VB 58S e 100S tiveram um pequeno aumento na sua cristalinidade. As análises de BET (adsorção de $\mathrm{N}_{2}$ ) mostraram estrutura mesoporosa no material. A área de superfície específica variou de $73,2 \mathrm{~m}^{2} / \mathrm{g}$ no scaffold $58 \mathrm{~S}$ tratado a $800{ }^{\circ} \mathrm{C}$ até 331,2 $\mathrm{m}^{2} / \mathrm{g}$ no scaffold $100 \mathrm{~S}$ tratado a $800{ }^{\circ} \mathrm{C}$. O material não é tóxico pelos ensaios MTT de citotoxicidade. Os resultados mostraram que é possível adicionar agentes porogênicos ao VB obtido via sol-gel e obter scaffolds favoráveis à regeneração tecidual óssea.
\end{abstract}

Palavras-chave: vidro bioativo, agente porogênico, biomateriais, sol-gel, cristalinidade.

\begin{abstract}
The use of biomaterials capable of generating a biological response has been one of the biggest progresses in regenerative medicine, due to their ability to support growth stimulation and damaged tissue regeneration. In this context, bioceramics, particularly bioactive glass $(B G)$, were the subject of many studies. The technique of porogen agent addition for the synthesis of scaffolds is an interesting procedure, because several types of porogen agents can be used. The aim of the present work was to obtain scaffolds using four porogen agents and to evaluate the effects that a change in treatment temperature can have on their crystallinity. Scaffolds of sol-gel bioactive glass $100 \mathrm{~S}\left(100 \% \mathrm{SiO}_{2}\right.$ ) using as porogen agents paraffin 1, paraffin 2, wax and CMC (carboxymethyl cellulose) were synthesized and characterized. As the best results were obtained with paraffin 1, scaffolds $58 \mathrm{~S}\left(60 \% \mathrm{SiO}_{2}-36 \% \mathrm{CaO}-4 \% \mathrm{P}_{2} \mathrm{O}_{5}\right)$ and $100 S$ using paraffin 1 as porogen agent were prepared. The scaffolds were submitted to different treatment temperatures to evaluate the effect on their crystallinity. Pore structure was analyzed by scanning electron microscopy and micro-computed tomography. Scaffolds presented satisfactory pore size and pore size distribution, important characteristics for scaffolds because they allow cell migration, nutrient transport, vascularization and tissue ingrowth. X-ray powder diffraction showed the amorphous nature of the scaffolds. At $900^{\circ} \mathrm{C}$, scaffolds BG $58 \mathrm{~S}$ and $100 \mathrm{~S}$ showed a small increase in crystallinity. BET analysis ( $\mathrm{N}_{2}$-adsorption) indicated a mesoporous structure. The specific surface area varied from $73.2 \mathrm{~m}^{2} / \mathrm{g}$ for scaffold $58 \mathrm{~S}$ treated at $800{ }^{\circ} \mathrm{C}$ to $331.2 \mathrm{~m}^{2} / \mathrm{g}$ for scaffold $100 \mathrm{~S}$ treated at $800^{\circ} \mathrm{C}$. The materials obtained showed no toxic effects by MTT cytotoxicity assays. Results showed that the development of scaffolds is possible using porogen agents, with $3 D$ interconnected porous structure and might therefore be a potential biomaterial for bone tissue regeneration.
\end{abstract}

Keywords: bioactive glass, porogen agent, biomaterials, sol-gel, crystallinity.

\section{INTRODUÇÃO}

A engenharia de tecidos e a medicina regenerativa têm como objetivo promover a cura e idealmente a regeneração das estruturas teciduais, restabelecendo a função tecidual com previsibilidade, mais rapidamente e de forma menos invasiva em relação às técnicas convencionais. Enxertos ósseos autógenos fornecem uma matriz osteocondutora, além 
de células e fatores de crescimento. Entretanto, a qualidade do osso autógeno é variada, dependendo da saúde geral do paciente. Muitos pacientes necessitam de um enxerto ósseo superior e em maior quantidade que seu organismo pode oferecer, tais como pacientes com osteopenia, osteoporose, diabetes e história de fumo [1].

Os vidros bioativos (VBs) são materiais que possuem capacidade de induzir uma resposta biológica na interface do material, levando à formação de camada de hidroxiapatita carbonatada (HCA), responsável pela formação de forte ligação entre o material e os tecidos moles e duros [2]. O conceito de materiais bioativos formarem ligação com o osso foi sugerido inicialmente por Hench e, desde então, esses materiais vêm sendo intensamente estudados em variadas composições para aplicações na área biomédica [3]. Os componentes principais dos VBs são $\mathrm{SiO}_{2}, \mathrm{CaO}, \mathrm{P}_{2} \mathrm{O}_{5} \mathrm{e}$ $\mathrm{Na}_{2} \mathrm{O}$ em proporções específicas. A composição do sistema é um fator determinante para a interação do biomaterial com o tecido, sendo que a mais comum é formada por $\mathrm{SiO}_{2}, \mathrm{CaO}$ e $\mathrm{P}_{2} \mathrm{O}_{5}$ [4]. O VB no fluido corpóreo está relacionado com as seguintes reações interfaciais: primeiro ocorre a troca de íons de $\mathrm{Na}^{+}$com íons de hidrogênio do fluido corpóreo e formação de ligação de $\mathrm{SiOH}$ e liberação de $\mathrm{Si}(\mathrm{OH})_{4}$; a policondensação de $\mathrm{SiOH}+\mathrm{SiOH} \rightarrow \mathrm{Si}-\mathrm{O}-\mathrm{Si}$ forma sílica gel hidratada. A adsorção de $\mathrm{Ca}^{2+}+\mathrm{PO}_{4}^{3-}+\mathrm{CO}_{3}^{2-}$ do $\mathrm{VB}$ pelo organismo receptor ocasiona a cristalização da hidroxiapatita carbonatada com adsorção de grupos biológicos na camada de hidroxiapatita e ação dos macrófagos. Nesse estágio ocorre a ligação de células indiferenciadas capazes de diferenciar em osteoblastos, as quais irão diferenciar e proliferar, gerando uma matriz; com a cristalização da matriz há a proliferação e o crescimento ósseo [5]. A arquitetura 3D do osso mineralizado é criada pelos osteoblastos, quando células são expostas a determinadas concentrações de constituintes iônicos solúveis liberados pelo VB. Aproximadamente 17 a 20 ppm de Si solúvel e 88 a 100 ppm de íons de Ca solúveis são necessários. A principal função dos VBs é liberar concentrações críticas de íons biologicamente ativos com taxas necessárias para proliferação e diferenciação celular [6-8]. O tratamento térmico e mudanças na composição dos VBs permitem controlar sua velocidade de reabsorção no organismo. $\mathrm{O}$ aumento do teor de silício diminui a taxa de degradação, sendo este um efeito direto da composição dos VBs. Tratamentos térmicos em elevadas temperaturas tornam esse biomaterial mais cristalino, diminuindo a resposta do organismo ao biomaterial $[9,10]$.
O scaffold ideal deve comandar a regeneração tecidual e remodelação in vivo; para tanto, deve ter uma superfície osteocondutora para que ocorra ligação e migração celular. Tem que induzir quimiotaxia (processo de locomoção de células em direção a um gradiente químico), estimular proliferação celular e coordenar a síntese de matriz no tecido hospedeiro. A complexidade da resposta celular para sinalização da matriz extracelular fez com que se desenvolvessem scaffolds tridimensionais (3D) que imitam as propriedades da matriz extracelular e não atuam apenas como suporte celular e tecidual [11]. Uma das maiores promessas de scaffolds para aplicação em engenharia de tecidos ósseos são os VBs, devido à bioatividade destes materiais. Várias técnicas permitem obter scaffolds altamente porosos, com estruturas em três dimensões, porosidade adaptada e tamanho de poro e interconectividade adequados [12]. Técnicas diversas existem para confeccionar scaffolds de VB, como a técnica de replicação da esponja [13, 14], técnica foaming (da espuma) de barbotinas cerâmicas [15], técnica conhecida como robocasting [16], também o desenvolvimento de scaffolds de VB por electrospun [17] e obtenção de scaffolds via adição de agentes porogênicos $[18,19]$. A técnica de adição de agentes porogênicos é uma técnica versátil; uma infinidade de agentes porogênicos pode ser utilizada e nenhum estudo foi encontrado na literatura sobre sua adição ao VB obtido via sol-gel. Apoiado ao grande potencial dos VBs, este estudo surge com os objetivos de: (i) desenvolver uma nova rota de obtenção de scaffolds porosos de VB por adição de agentes porogênicos ao VB obtido via sol-gel; e (ii) avaliar os efeitos provocados na sua cristalinidade por modificações na composição e mudanças nas temperaturas de tratamento dos scaffolds de VB obtidos.

\section{MATERIAIS E MÉTODOS}

Síntese dos scaffolds com $100 \% \mathrm{SiO}_{2}$ com adição de diferentes agentes porogênicos

Os scaffolds de VB foram confeccionados pela rota sol-gel [20, 21], a princípio com composição $100 \%$ de sílica (VB100S). Os reagentes foram: alcóxido tetraetilortossilicato (TEOS) - Aldrich, 98\% de pureza, $\mathrm{Si}\left(\mathrm{OC}_{2} \mathrm{H}_{5}\right)_{4}$, precursor de $\mathrm{SiO}_{2}$, água deionizada, ácido nítrico $2 \mathrm{~N}$ - Synth e solução $5 \% \mathrm{v} / \mathrm{v}$ ácido fluorídrico HF - Merck. A síntese seguiu o método sol-gel, rota de alcóxido, com a seguinte metodologia para o vidro 100S:

Tabela I - Composições das misturas (\% em massa) utilizadas para síntese de scaffold 100S, para seleção do agente porogênico. [Table I - Mixed composition (wt\%) used for the synthesis of the scaffold 100S, for porogen agent selection.]

\begin{tabular}{ccccc}
\hline Amostra & Agente porogênico & \% de sol & \% de agente porogênico & \% de HF (v/v do sol) \\
\hline VBC & Cera & 40 & 60 & 5 \\
VBP1 & Parafina-1 & 45 & 55 & 5 \\
VBP2 & Parafina-2 & 30 & 70 & 5 \\
VBCMC & Parafina-1 + CMC & 27,4 & 50,3 de parafina-1 & 5 \\
& & & 22,3 de CMC & 5 \\
\hline
\end{tabular}


220,4 mL de água deionizada, $36,7 \mathrm{~mL}$ de solução $2 \mathrm{~N}$ de ácido nítrico $\left(\mathrm{HNO}_{3}\right)$ e $228,6 \mathrm{~mL}$ de tetraetilortossilicato (TEOS) foram adicionados em um béquer e a agitação foi mantida por $60 \mathrm{~min}$. $\mathrm{O}$ sol resultante foi misturado com o agente porogênico, conforme a Tabela I, juntamente com o $\mathrm{HF}$, sendo este $5 \%(\mathrm{v} / \mathrm{v})$ do sol, e colocado em recipientes de teflon fechados hermeticamente com tampa lisa e porcas de teflon. A quantidade de agente porogênico adicionada foi determinada pela máxima porcentagem dispersa do agente porogênico no biovidro sol-gel. Esses foram colocados na estufa para gelificação e envelhecimento a $60{ }^{\circ} \mathrm{C}$ por $72 \mathrm{~h}$. Em seguida iniciou-se a secagem. As tampas foram trocadas por tampas com furos e fechadas com as porcas de teflon. Os recipientes foram colocados na estufa a $60^{\circ} \mathrm{C}$ e a temperatura foi elevada em $10^{\circ} \mathrm{C}$ a cada $24 \mathrm{~h}$ até atingir a temperatura de $120^{\circ} \mathrm{C}$ e mantida por mais $24 \mathrm{~h}$. Os scaffolds foram tratados termicamente nas temperaturas $700,800 \mathrm{e}$ $900{ }^{\circ} \mathrm{C} \mathrm{com}$ taxa de aquecimento de $1^{\circ} \mathrm{C} / \mathrm{min}$, por $1440 \mathrm{~min}$, para que degradasse toda a fase orgânica.

Síntese dos scaffolds de diferentes composições com adição do agente porogênico parafina 1

As análises dos resultados obtidos com diferentes agentes porogênicos, considerando integridade das amostras, tamanho e distribuição de tamanho de poros, permitiu selecionar o agente porogênico parafina 1 para a continuidade desse estudo. Vidros bioativos de duas composições foram então sintetizados: VB58S $\left(58 \% \mathrm{SiO}_{2}-\right.$ $33 \%$ CaO-9\% $\mathrm{P}_{2} \mathrm{O}_{5}$ em porcentagem mássica) e VB100S $\left(100 \% \mathrm{SiO}_{2}\right)$. Para o VB58S foram usados os seguintes reagentes: 132,2 $\mathrm{mL}$ de água deionizada, 22,04 $\mathrm{mL}$ de ácido nítrico $2 \mathrm{~N}$ - Synth, $13,75 \mathrm{~mL}$ de trietilfosfato (TEP) - Aldrich, $137,1 \mathrm{~mL}$ de tetraetilortossilicato (TEOS) - Aldrich e 85,01 $\mathrm{g}$ de nitrato de cálcio $\left[\mathrm{Ca}\left(\mathrm{NO}_{3}\right)_{2} \cdot 4 \mathrm{H}_{2} \mathrm{O}\right]$ - Synth. Foram adicionados em um béquer água deionizada e ácido nítrico. Em seguida foram adicionados o TEOS e TEP e mantida a agitação por $60 \mathrm{~min}$. O nitrato de cálcio foi adicionado aos poucos à mistura; a agitação foi mantida por mais $30 \mathrm{~min}$. Para o VB100S usou-se: $220,4 \mathrm{~mL}$ de água deionizada, 36,7 $\mathrm{mL}$ de ácido nítrico $2 \mathrm{~N}\left(\mathrm{HNO}_{3}\right)$ - Synth, e $228,6 \mathrm{~mL}$ de tetraetilortossilicato (TEOS) - Aldrich. Foram adicionados em um béquer água deionizada e ácido nítrico. Em seguida foi adicionado o TEOS e mantida a agitação por $60 \mathrm{~min}$. $\mathrm{O}$ sol resultante foi misturado com o agente porogênico (50\% de massa por volume para o $58 \mathrm{~S}$ e $55 \%$ de massa por volume para o 100S) e, então, foram adicionados $5 \%$ de HF - Merck (v/v do sol, como catalisador de gelificação). A mistura foi colocada em recipientes de teflon, que foram fechados hermeticamente com a tampa lisa e porcas de teflon e, então, colocados na estufa para gelificação e envelhecimento a $60^{\circ} \mathrm{C}$ por $72 \mathrm{~h}$. Para a secagem as tampas foram trocadas por tampas com furos e fechadas com as porcas de teflon. Os recipientes foram colocados na estufa a $60{ }^{\circ} \mathrm{C}$ e a temperatura foi elevada em $10^{\circ} \mathrm{C}$ a cada $24 \mathrm{~h}$ até atingir a temperatura de $120^{\circ} \mathrm{C}$ e mantida por mais $24 \mathrm{~h}$. Os scaffolds (VB58S e VB100S) obtidos após secagem foram colocados para tratamento térmico a 700,800 e $900{ }^{\circ} \mathrm{C}$ com taxa de aquecimento de $1{ }^{\circ} \mathrm{C} / \mathrm{min}$ por $1440 \mathrm{~min}$.

\section{Caracterização estrutural dos scaffolds}

A morfologia e composição química dos scaffolds foram analisadas por microscopia eletrônica de varredura (MEV) e por espectroscopia de energia dispersiva de raios X (EDS), respectivamente, utilizando o equipamento FEI-INSPECT S50, operado a $20 \mathrm{kV}$ e equipado com um espectrômetro de energia dispersiva (EDS) da EDAX Genesis. Foi realizada a difração de raios X (DRX) no equipamento PW9710mpd, Philips, usando radiação CuK $\alpha(\lambda=1,54056 \AA$ Á) a $40 \mathrm{kV}$ e $30 \mathrm{~mA}$. A análise foi conduzida com $2 \theta$ variando de $3,00^{\circ}$ a $90,00^{\circ}$ com passo de $0,06^{\circ}$ e tempo de coleta de $1 \mathrm{~s}$. A microtomografia computadorizada $(\mu \mathrm{CT})$ permitiu avaliar a morfologia, tamanho, distribuição e interconectividade dos poros, e foi realizada com aparelho Skyscan 1174 (Aartselaar), equipado com câmera de 1,3 MP, utilizandose fonte de $50 \mathrm{kV}$ e corrente de $800 \mu \mathrm{A}$ com resolução de $14 \mu \mathrm{m}$. Foi utilizado filtro de alumínio com $1 \mathrm{~mm}$ de espessura. As amostras foram fixadas em um suporte e rotacionadas $180^{\circ}$, com imagens adquiridas a cada $0,7^{\circ}$. Após o escaneamento, cortes transversais foram reconstruídos em estruturas tridimensionais utilizando-se software SkyScan. As análises por adsorção de nitrogênio, BET (por isotermas de Brunauer, Emmett e Taller), foram realizadas utilizando um equipamento Quantachrome NovaWin, v. 10.01, e permitiram a determinação da área superficial total, o volume de poros $\mathrm{e}$ a distribuição de meso e microporos.

\section{Avaliação da citotoxidade dos scaffolds}

A citotoxicidade do scaffold foi avaliada pelo ensaio de MTT [brometo de 3-(4,5-dimetiltiazol-2-il)-2,5difeniltetrazólio], que analisa quantitativamente a redução do sal de tetrazólio (MTT) pelo complexo enzimático piruvato desidrogenase presente nas mitocôndrias. A formação de um produto final de cristais de formazan é mensurada em espectrofotômetro a $595 \mathrm{~nm}$. Esse ensaio é usado especificamente para avaliar a funcionalidade mitocondrial e proliferação celular.

Os ratos Wistar neonatos (1 a 5 dias), fêmeas, utilizados para este estudo foram obtidos a partir do Centro de Bioterismo da Universidade Federal de Minas Gerais (CEBIO). O estudo foi em conformidade com o Manual sobre Cuidados e Uso de Animais de Laboratório publicado pelo National Institutes of Health dos EUA (NIH publicação ${ }^{\circ}$ 85-23, revista em 1996). Os osteoblastos foram extraídos de calvária de 5 ratos neonatos. Os animais foram sacrificados e suas calvárias foram retiradas e cortadas em fragmentos de $2 \mathrm{~mm} / 2 \mathrm{~mm}$; esses fragmentos foram colocados em um tubo falcon contendo tripsina $1 \%$ e levados para estufa a $37^{\circ} \mathrm{C}$ e $5 \%$ de $\mathrm{CO}_{2}$ por $15 \mathrm{~min}$. O sobrenadante foi descartado e foi colocado 0,06 g de colagenase tipo $2 \mathrm{em} 30 \mathrm{~mL}$ de DMEM sem soro e colocado por $30 \mathrm{~min}$ a $37^{\circ} \mathrm{C}$. O sobrenadante foi descartado e foi colocado mais DMEM e colagenase 
e retornado para estufa por mais 20 min. O tubo falcon com o material foi centrifugado por $5 \mathrm{~min}$ a $1400 \mathrm{rpm}$. O sobrenadante foi recolhido e colocado na garrafa de cultura T25 com $5 \mathrm{~mL}$ de meio DMEM complementado com soro fetal bovino (SFB). Foram feitas mais 2 digestões de $20 \mathrm{~min}$. Os osteoblastos da segunda passagem foram tripsinizados e plaqueados ( $3 \times 10^{4}$ células/poço) em placas de 24 poços. Após $24 \mathrm{~h}$, o meio foi aspirado, colocado um meio de cultivo fresco e as populações celulares foram expostas a 1 $\mathrm{mg} / \mathrm{mL}$ de biomaterial [22]. O método de esterilização foi radiação ultravioleta. Como controle positivo, foi utilizado Triton X-100 a 1\%. Como controle negativo, foi utilizado meio de cultivo DMEM complementado com SFB. Todos os ensaios foram realizados em triplicata $(n=3)$. Após $24 \mathrm{~h}$ de exposição, todo o meio foi aspirado, sendo colocados $240 \mu \mathrm{L}$ de meio de cultura complementado com SFB em cada poço. Foram acrescentados $170 \mu \mathrm{L}$ de MTT $(5 \mathrm{mg} / \mathrm{mL})$ em cada poço, seguido por uma incubação de $4 \mathrm{~h}$ a $37^{\circ} \mathrm{Ce} 5 \%$ de $\mathrm{CO}_{2}$. Em seguida foram colocados $170 \mu \mathrm{L}$ de solução de SDS/10\% $\mathrm{HCl}$. A placa foi incubada a $37{ }^{\circ} \mathrm{C}$ e $5 \%$ de $\mathrm{CO}_{2}$ por $18 \mathrm{~h}$. Então, foram retirados $100 \mu \mathrm{L}$ de cada poço e transferidos para uma placa plana de 96 poços e a quantificação foi feita em espectrofotômetro (ADAP 1.6, Anthos Labtec Instruments) com filtro de $595 \mathrm{~nm}$ (análise estatística - Oneway / Bonferroni / GraphPad Prism).

\section{RESULTADOS E DISCUSSÃO}

\section{Scaffolds obtidos com diferentes agentes porogênicos}

Os agentes porogênicos foram analisados por MEV para avaliação de seus tamanhos e formatos. A cera apresentou formato disforme e variação de tamanho de 9 a $204 \mu \mathrm{m}$. O CMC mostrou forma alongada, com distribuição de tamanho de 28 a $382 \mu \mathrm{m}$. A parafina 1 apresentou formato mais arredondado com distribuição de tamanho de 609 a $993 \mu \mathrm{m}$. A parafina 2 mostrou formato arredondado e mais uniforme, com distribuição de tamanho de 1,9 a 2,3 mm. Os scaffolds produzidos com adição de CMC e parafina desintegraramse durante o tratamento térmico. Assim, não foi possível obter amostras viáveis a partir dessa composição de agentes porogênicos. O VBC $700{ }^{\circ} \mathrm{C}$ apresentou fragilidade ao manuseio, formato disforme e com o aumento de temperatura de tratamento térmico os scaffolds tornaram-se mais fáceis de manusear. O VBC $900{ }^{\circ} \mathrm{C}$ mostrou maior facilidade de manuseio e, visualmente, formato mais uniforme que os VBC $700{ }^{\circ} \mathrm{C}$ e VBC $800{ }^{\circ} \mathrm{C}$. Os VBP $1700{ }^{\circ} \mathrm{C}$, VBP1 $800{ }^{\circ} \mathrm{C}$ e VBP $1900{ }^{\circ} \mathrm{C}$ demonstraram comportamento ao manuseio e formato mais uniforme que os demais scaffolds; o VBP $1700{ }^{\circ} \mathrm{C}$ com formato menos uniforme e com maior dificuldade de manusear, apresentou maior facilidade de fratura que os VBP1 $800{ }^{\circ} \mathrm{C}$ e VBP1 $900{ }^{\circ} \mathrm{C}$. Os VBP2 $700{ }^{\circ} \mathrm{C}, \mathrm{VBP} 2800{ }^{\circ} \mathrm{C}$ e VBP2 $900{ }^{\circ} \mathrm{C}$ apresentaram baixa resistência ao manuseio, com maior dificuldade de manuseálos, em relação aos demais scaffolds. As análises de MEV dos VBC 700, 800 e $900{ }^{\circ} \mathrm{C}$ mostraram poucos poros abertos, sendo o diâmetro de 42 a $102 \mu \mathrm{m}, 55 \mu \mathrm{m}$ e 33 a
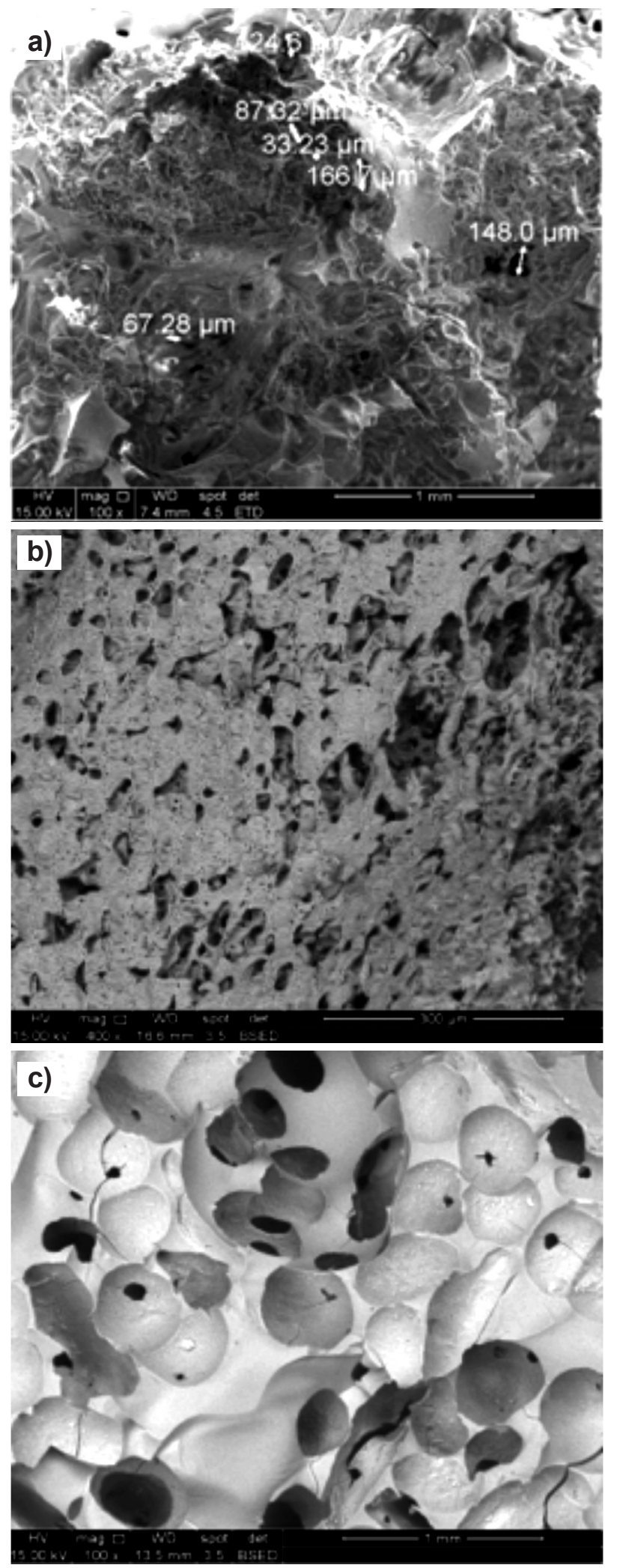

Figura 1: Micrografias obtidas por microscopia eletrônica de varredura dos scaffolds produzidos com adição de cera, parafina 2 e parafina 1 tratados termicamente a: (a) $\mathrm{VBC} 900{ }^{\circ} \mathrm{C}$, (b) VBP2 $800{ }^{\circ} \mathrm{C}$, (c) VBP $1700{ }^{\circ} \mathrm{C}$.

[Figure 1: SEM micrographs of the scaffolds produced with addition of wax, paraffin 2 and paraffin 1 heat treated at: (a) VBC $900{ }^{\circ} \mathrm{C}$, (b) VBP2 $800{ }^{\circ} \mathrm{C}$, (c) VBP1 $700{ }^{\circ} \mathrm{C}$.] 
$167 \mu \mathrm{m}$, respectivamente. As amostras deVBP1 700, 800, $900{ }^{\circ} \mathrm{C}$ mostraram-se, nas imagens de MEV, porosas com diâmetro de poros de 113 a 1174 um, 219 a 1041 um e 64 a $595 \mu \mathrm{m}$, respectivamente. As amostras de VBP2 700, $800 \mathrm{e}$ $900{ }^{\circ} \mathrm{C}$ mostraram-se no MEV com poros muito grandes e abertos, sendo que o tamanho de poro variou de $437 \mu \mathrm{m}$, no scaffold tratado a $900{ }^{\circ} \mathrm{C}$, até $1045 \mu \mathrm{m}$, no scaffold tratado a $800^{\circ} \mathrm{C}$. A Fig. 1 ilustra as imagens de MEV dos scaffolds. Os espectros de EDS de todas as amostras mostraram a presença de silício e oxigênio.

Os scaffolds produzidos com adição de cera, parafina 1 e parafina 2 foram submetidos a análise por $\mu \mathrm{CT}$, que mostraram tamanhos médios de poros muito superiores aos observados por MEV; para o VBC tratado a 700, 800 e $900{ }^{\circ} \mathrm{C}$ foram 419,411 e $399 \mu \mathrm{m}$, respectivamente. Isso se explica devido a $\mu \mathrm{CT}$ avaliar o volume do scaffold em 3D, além de outras razões variadas, como contração da amostra no MEV, especificações de claro e escuro na $\mu \mathrm{CT}$, entre outros. A $\mu \mathrm{CT}$ para o VBP1 tratado a 700, 800 e $900{ }^{\circ} \mathrm{C}$ resultou em diâmetros de poros de 442, 400 e $371 \mu \mathrm{m}$, respectivamente, e a $\mu \mathrm{CT}$ do VBP2 tratado a 700, 800 e $900{ }^{\circ} \mathrm{C}$ mostrou diâmetros de poros de 532, 599 e $594 \mu \mathrm{m}$, respectivamente, e, devido ao grande tamanho e quantidade dos poros, os scaffolds apresentaram menor resistência ao manuseio em relação aos scaffolds produzidos com parafina 1 e cera. Todas as amostras apresentaram quantidade de poros abertos superior a $50 \%$, exceto o
VBC $900{ }^{\circ} \mathrm{C}$ que apresentou $44 \%$ de porosidade. Ressaltase que foram calculados por $\mu \mathrm{CT}$ apenas os macroporos, sendo a porosidade total provavelmente maior do que os resultados obtidos nesta caracterização, uma vez que os materiais obtidos pela rota sol-gel apresentam também um grande volume de mesoporos. Os scaffolds produzidos, além de terem quantidade satisfatória de poros abertos (superior a 50\%), têm uma distribuição de tamanho de poros (superior a $150 \mu \mathrm{m}$ ) condizente para regeneração óssea [23]. As imagens de $\mu \mathrm{CT}$ mostram as diferenças estruturais em 3D dos VBC, VBP1 e VBP2 (Fig. 2), e a Fig. 3 apresenta o corte em 2D dos VBC, VBP1 e VBP2 tratados a $700{ }^{\circ} \mathrm{C}$. $\mathrm{O}$ VBC $700{ }^{\circ} \mathrm{C}$ apresentou $68 \%$ de poros abertos e os poros foram interconectados, mas irregulares, o que condiz com o formato do agente porogênico, com uma ampla distribuição de tamanho, sendo o diâmetro médio de $419 \mu \mathrm{m}$. O VBP1 $700{ }^{\circ} \mathrm{C}$ apresentou $67,4 \%$ de poros abertos com formato mais uniforme, condizente com o formato do agente porogênico; os poros foram interconectados e com distribuição de tamanho de poros satisfatória, com diâmetro médio de $442 \mu \mathrm{m}$. O VBP2 $700{ }^{\circ} \mathrm{C}$ apresentou $66 \%$ de poros abertos, grandes, arredondados, condizentes com o formato do agente porogênico, os poros foram inteconectados e o diâmetro médio foi de $532 \mu \mathrm{m}$.

As distribuições de tamanho de poros obtidas para os scaffolds VBC $700{ }^{\circ} \mathrm{C}$, VBP1 $700{ }^{\circ} \mathrm{C}$ e VBP2 $700{ }^{\circ} \mathrm{C}$ são mostradas na Fig. 4, que apresentaram médias de
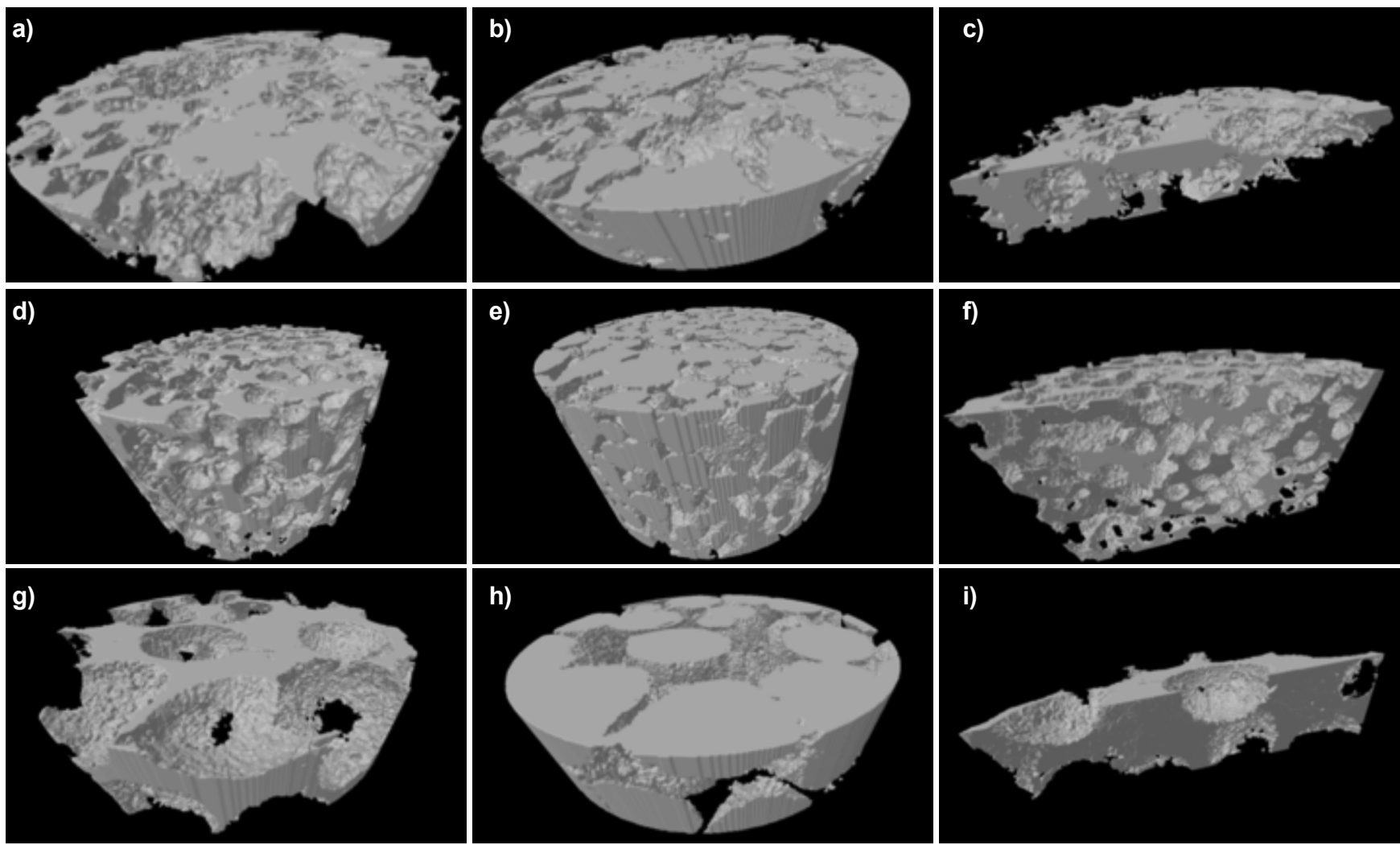

Figura 2: Imagens 3D de $\mu \mathrm{CT}$ de amostras tratadas a $700{ }^{\circ} \mathrm{C}$ de (a-c) VBC, (d-f) VBP1 e (g-i) VBP2: (a,d,g) - scaffold; (b,e,h) - estrutura de poros; (c,f,i) - estrutura interna.

[Figure 2: $3 D \mu C T$ images of samples treated at $700{ }^{\circ} \mathrm{C}$ of $(a-c)$ VBC, (d-f) VBP1, and (g-i) VBP2: (a,d,g) - scaffold; (b,e,h) - pores structure; $(c, f, i)$ - internal structure.] 
tamanho de poros de 419, 442 e $532 \mu \mathrm{m}$, respectivamente. A parafina 1 como agente porogênico permitiu a formação de scaffolds com melhor distribuição de tamanho de poros; as caracterizações também permitiram observar que nos scaffolds VBP1 os poros foram mais interconectados, prérequisitos para que aconteça a regeneração óssea. A Tabela II resume os dados obtidos através das caracterizações estruturais dos scaffolds VBC, VBP1 e VBP2.
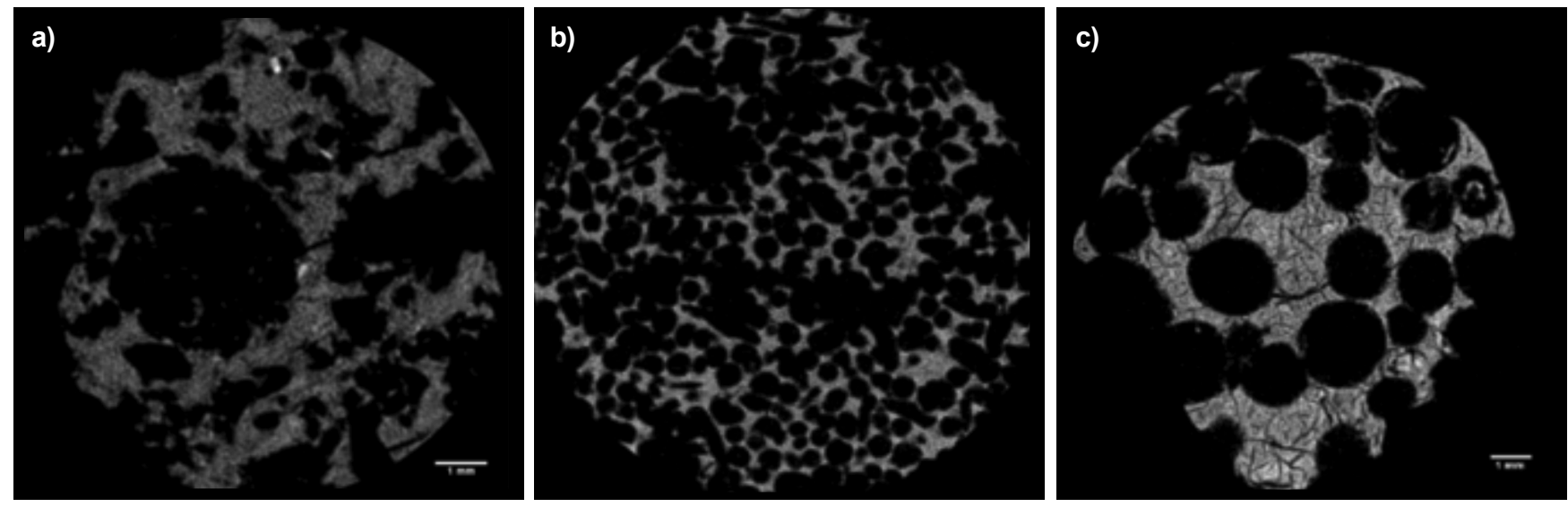

Figura 3: Imagens de corte 2D de $\mu \mathrm{CT}$ de amostras tratadas a $700{ }^{\circ} \mathrm{C}$ de (a) VBC, (b) VBP1 e (c) VBP2.

[Figure 3: $2 D$ section $\mu C T$ images of samples treated at $700{ }^{\circ} \mathrm{C}$ of (a) $\mathrm{VBC}$, (b) VBP1, and (c) VBP2.]
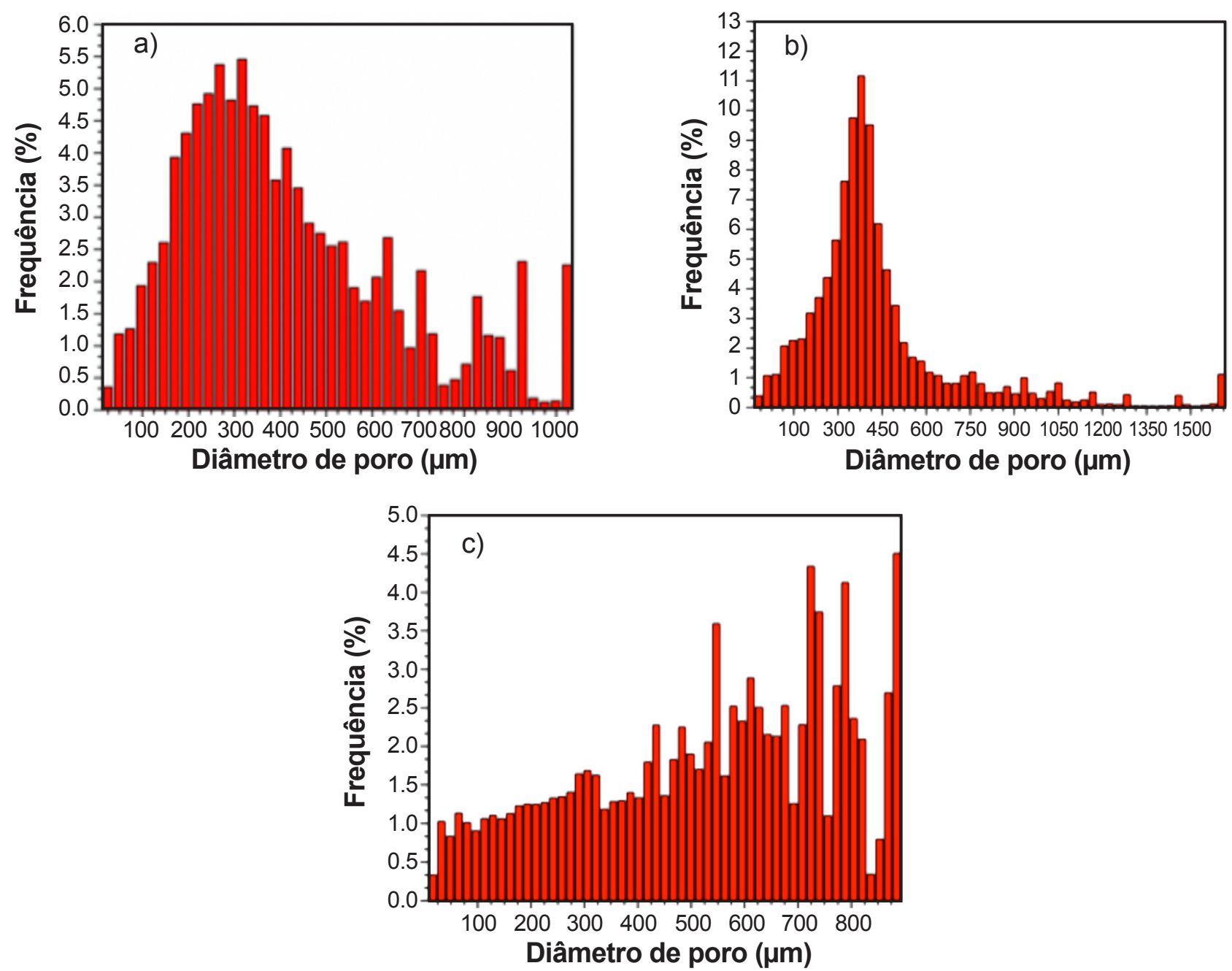

Figura 4: Distribuições de tamanho de poros obtidas por $\mu \mathrm{CT}$ : (a) VBC $700{ }^{\circ} \mathrm{C}$, (b) VBP1 $700{ }^{\circ} \mathrm{C}$ e (c) $\mathrm{VBP} 2700{ }^{\circ} \mathrm{C}$. [Figure 4: Pore size distributions obtained by $\mu \mathrm{CT}$ : (a) $\mathrm{VBC} 700^{\circ} \mathrm{C}$, (b) $\mathrm{VBP} 1700^{\circ} \mathrm{C}$, and (c) $\mathrm{VBP} 2700^{\circ} \mathrm{C}$.] 
Tabela II - Diâmetro e fração de poros abertos (obtidos por $\mu \mathrm{CT}$ e MEV) dos scaffolds de VB100S com adição dos agentes porogênicos: cera (C), parafina $1(\mathrm{P} 1)$ e parafina $2(\mathrm{P} 2)$, tratados a 700,800 e $900{ }^{\circ} \mathrm{C}$.

[Table II - Open pore diameter and fraction (obtained by $\mu$ CT and SEM) for the scaffolds VB100S with addition of porogen agents: wax $(C)$, paraffin $1(P 1)$, and paraffin $2(P 2)$, treated at 700,800 and $\left.900{ }^{\circ} \mathrm{C}.\right]$

\begin{tabular}{|c|c|c|c|c|}
\hline Scaffold & $\begin{array}{c}\% \text { total de poros por } \\
\mu \mathrm{CT}\end{array}$ & $\begin{array}{c}\% \text { de poros abertos por } \\
\mu \mathrm{CT}\end{array}$ & $\begin{array}{l}\text { Diâmetro dos poros por MEV } \\
\qquad(\mu \mathrm{m})\end{array}$ & $\begin{array}{l}\text { Diâmetro dos poros por } \mu \mathrm{CT} \\
\qquad(\mu \mathrm{m})\end{array}$ \\
\hline $\mathrm{VBC} 700{ }^{\circ} \mathrm{C}$ & 72,0 & 68,0 & 42 a 102 & 419 \\
\hline $\mathrm{VBC} 800{ }^{\circ} \mathrm{C}$ & 74,0 & 72,0 & 55 & 411 \\
\hline VBC $900{ }^{\circ} \mathrm{C}$ & 57,0 & 44,0 & 33 a 167 & 399 \\
\hline VBP1 $700^{\circ} \mathrm{C}$ & 67,4 & 67,4 & 113 a 1174 & 442 \\
\hline VBP1 $800^{\circ} \mathrm{C}$ & 67,6 & 67,6 & 219 a 1041 & 400 \\
\hline VBP1 $900^{\circ} \mathrm{C}$ & 70,0 & 70,0 & 64 a 595 & 371 \\
\hline VBP2 $700{ }^{\circ} \mathrm{C}$ & 66,0 & 66,0 & 1000 & 532 \\
\hline VBP2 $800^{\circ} \mathrm{C}$ & 79,0 & 79,0 & 756 a 1045 & 599 \\
\hline VBP2 $900^{\circ} \mathrm{C}$ & 66,0 & 66,0 & 438 a 989 & 594 \\
\hline
\end{tabular}

\section{Scaffolds VB58S e VB100S obtidos com parafina 1}

Todas as amostras produzidas com parafina 1 apresentaram resistência ao manuseio. O tamanho dos poros, de acordo com o MEV, diminuiu com o aumento de temperatura de tratamento térmico, em todos os scaffolds. A Fig. 5 mostra imagens macroscópica e de MEV do VBP158S- $800{ }^{\circ} \mathrm{C}$. A análise de EDS, de todas as amostras, confirmou a presença de cálcio e fósforo, além da sílica nos scaffolds, importantes para o aumento da bioatividade do scaffold.

A $\mu \mathrm{CT}$ permitiu calcular porcentagem e tamanho dos poros dos scaffolds através de análises tridimensionais realizadas em software. Deve ser considerado que o cálculo de porosidade considerou apenas os macroporos, sendo a porosidade total provavelmente maior. A quantidade de poros abertos e porosidade total diminuíram com o aumento de temperatura de tratamento nos scaffolds de VB58S; nesses scaffolds o aumento de temperatura de tratamento refletiu na diminuição da quantidade de poros, tornando as partículas mais densas e com número pouco menor de poros abertos; nos scaffolds de VB100S o aumento de temperatura não interferiu na porcentagem de poros abertos, mas o efeito do tratamento térmico fez com que diminuísse o tamanho dos poros. Pela análise de $\mu \mathrm{CT}$ do VBP158S-700 ${ }^{\circ} \mathrm{C}$ obteve-se

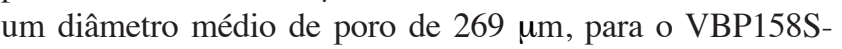
$800{ }^{\circ} \mathrm{C}$ o diâmetro de poros foi de $290 \mu \mathrm{m}$ e do VBP158S$900{ }^{\circ} \mathrm{C}$ de $278 \mu \mathrm{m}$. Para o VBP1100S-700, 800 e $900{ }^{\circ} \mathrm{C}$ os tamanhos médios de poros foram de 442,400 e $371 \mu \mathrm{m}$, respectivamente. Pelas análises de $\mu \mathrm{CT}$, foi possível avaliar o volume, a distribuição, a porcentagem de poros abertos, bem como a interconectividade dos poros. Os scaffolds apresentaram quase totalidade de poros abertos, quando se avalia volume total de poros/volume de poros abertos. A Fig. 6 mostra imagens de $\mu \mathrm{CT}$ do VBP158S-700 ${ }^{\circ} \mathrm{C}$ em 3D mostrando características estruturais do scaffold e rede de poros. Os scaffolds apresentaram tamanho médio de poros satisfatórios, de $269 \mu \mathrm{m}$. A porosidade superior a $50 \%$ e

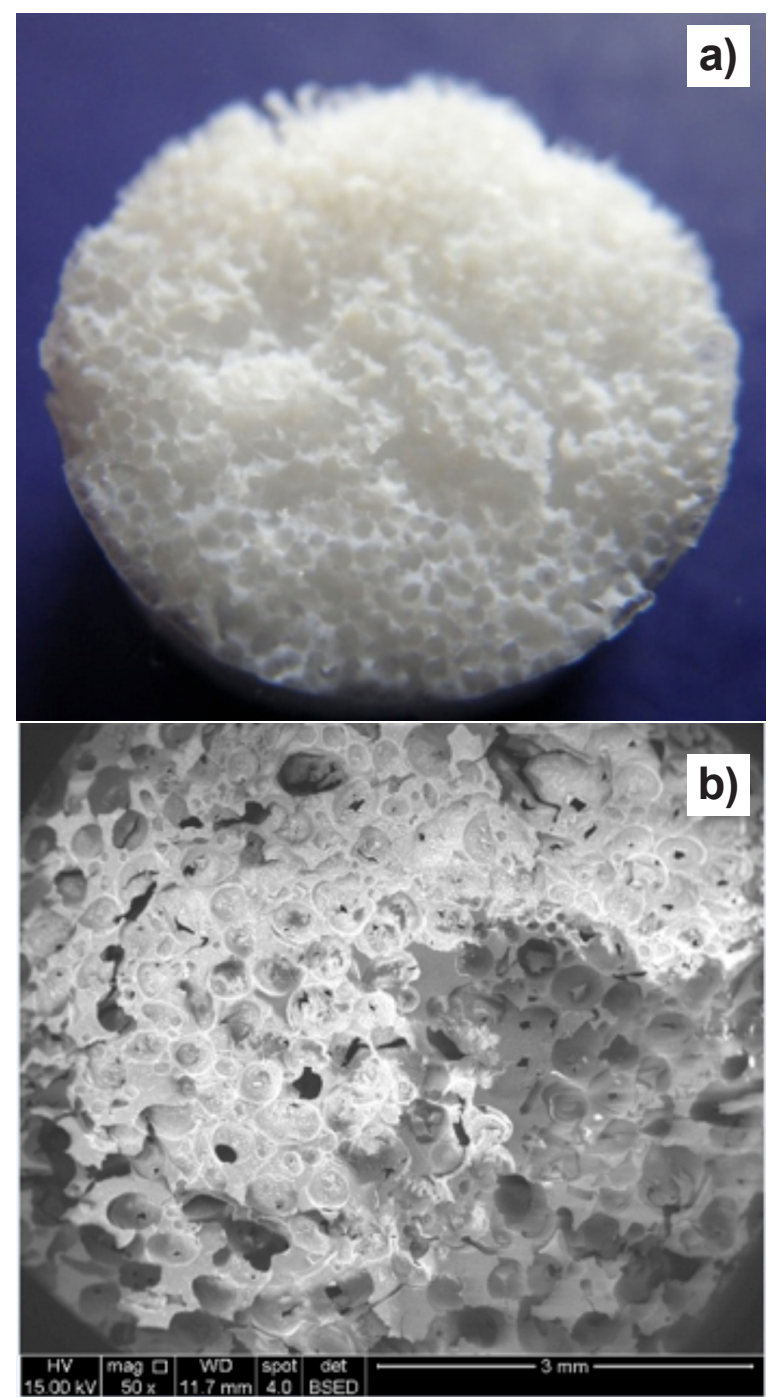

Figura 5: Imagens macroscópica (a) e de MEV (b) do VBPI58S- $800^{\circ} \mathrm{C}$.

[Figure 5: Macroscopic (a) and SEM (b) images of VBPI58S- $\left.800^{\circ} \mathrm{C}.\right]$ 
poros maiores que $150 \mu \mathrm{m}$ e interconectados são fatores importantes, pois permitem migração celular, transporte de nutrientes, vascularização e crescimento tecidual [24-27]. O VB100S com adição de parafina 1 apresentou porosidade superior a $67 \%$, com tamanho de poro suficiente para regeneração óssea. $\mathrm{O}$ aumento de temperatura ocasionou uma diminuição no tamanho dos poros. A Tabela III resume todos os dados obtidos nas análises estruturais dos scaffolds VB58S e VB100S com adição do agente porogênico parafina 1.
A Fig. 7 apresenta os resultados de DRX dos scaffolds doVB58S e 100S com adição de parafina 1 e tratamento térmico a 700,800 e $900{ }^{\circ} \mathrm{C}$. Observam-se poucos picos de cristalinidade, sendo que a definição dos picos indicaria a cristalização do material, obtendo possivelmente um material mais denso, mais resistente e menos bioativo. Os difratogramas de raios $\mathrm{X}$ de todas as amostras foram característicos de material amorfo, sem diferenças significativas em relação a cristalinidade. O resultado obtido é diferente do observado em outros trabalhos, como
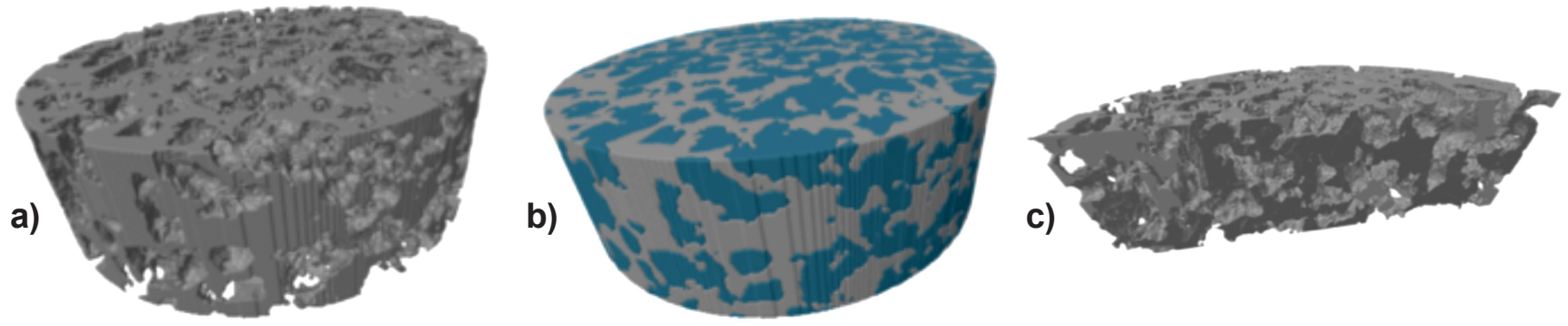

Figura 6: Imagens de $\mu \mathrm{CT}$ do VBPI58S-700 ${ }^{\circ} \mathrm{C}$ de (a) superfície do scaffold, (b) estrutura de poros e (c) estrutura interna. [Figure 6: $\mu$ CT images of VBPI58S-700 ${ }^{\circ} \mathrm{C}$ of $($ a $)$ scaffold surface, (b) pores structure, and (c) internal structure.]

Tabela III - Diâmetro e porcentagem de poros abertos obtidos por MEV e $\mu$ CT dos scaffolds de VB58S e VB100S com adição do agente porogênico parafina 1 (P1).

[Table III - Open pore diameter and fraction obtained by SEM and $\mu$ CT for the scaffolds $58 S$ and 100S with addition of porogen agent paraffin $1(P 1)$.]

\begin{tabular}{ccccc}
\hline Scaffolds & $\begin{array}{c}\text { \% de porosidade } \\
\text { total }\end{array}$ & $\begin{array}{c}\text { \% de poros } \\
\text { abertos }\end{array}$ & $\begin{array}{c}\text { Diâmetro dos poros por } \\
\text { MEV }(\mu \mathrm{m})\end{array}$ & $\begin{array}{c}\text { Média de diâmetro dos poros } \\
\text { por } \mu \mathrm{CT}(\mu \mathrm{m})\end{array}$ \\
\hline $\mathrm{VBP} 158 \mathrm{~S}-700{ }^{\circ} \mathrm{C}$ & 56,5 & 56,4 & 213 a 502 & 269 \\
$\mathrm{VBP} 158 \mathrm{~S}-800^{\circ} \mathrm{C}$ & 52,0 & 52,0 & 192 a 430 & 290 \\
$\mathrm{VBP} 158 \mathrm{~S}-900{ }^{\circ} \mathrm{C}$ & 51,0 & 51,0 & 112 a 454 & 278 \\
$\mathrm{VBP} 1100 \mathrm{~S}-700{ }^{\circ} \mathrm{C}$ & 67,4 & 67,4 & 113 a 1174 & 442 \\
$\mathrm{VBP} 1100 \mathrm{~S}-800{ }^{\circ} \mathrm{C}$ & 67,6 & 67,6 & 219 a 1041 & 400 \\
VBP1100S-900 ${ }^{\circ} \mathrm{C}$ & 70,0 & 70,0 & 64 a 595 & 371 \\
\hline
\end{tabular}
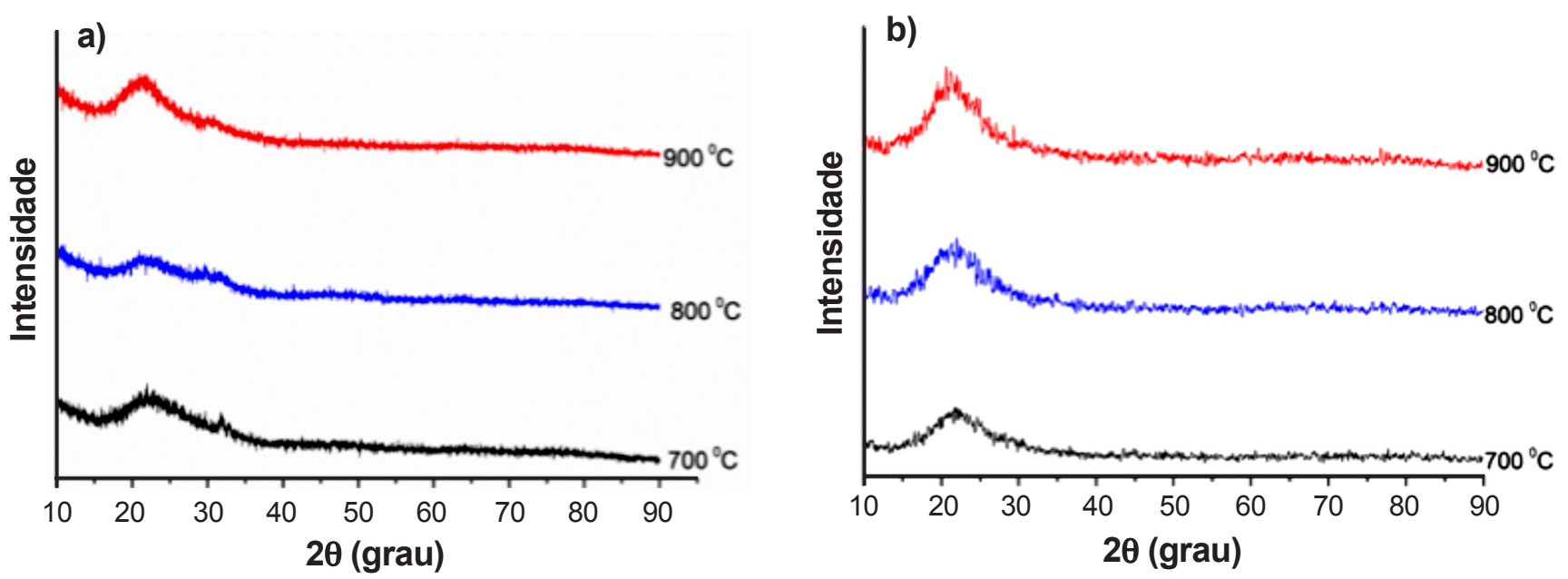

Figura 7: Difratogramas de raios X das amostras de scaffolds de (a) VB58S e (b) VB100 tratadas em diferentes temperaturas.

[Figure 7: XRD patterns of the scaffold samples of (a) VB58S and (b) VB100 treated at different temperatures.] 
Tabela IV - Área superficial específica (SSA) e volume e diâmetro de poros, determinados por BET - adsorção de nitrogênio, dos scaffolds de VB58S e VB100S com adição do agente porogênico parafina 1 (P1).

[Table IV - Specific surface area (SSA), and pore volume and diameter, determined by BET - nitrogen adsorption, of the scaffolds of VB58S and VB100S with addition of porogen agent paraffin 1 (P1).]

\begin{tabular}{cccc}
\hline Amostra & $\mathrm{SSA}\left(\mathrm{m}^{2} / \mathrm{g}\right)$ & Volume de poro $\left(\mathrm{cm}^{3} / \mathrm{g}\right)$ & Diâmetro de poro $(\mathrm{nm})$ \\
\hline VBP158S-700 ${ }^{\circ} \mathrm{C}$ & 325,5 & 1,1 & 13,6 \\
VBP158S-800 ${ }^{\circ} \mathrm{C}$ & 73,2 & 0,4 & 21,7 \\
VBP158S-900 ${ }^{\circ} \mathrm{C}$ & 100,6 & 0,3 & 13,1 \\
VBP1100S-700 ${ }^{\circ} \mathrm{C}$ & 261,4 & 1,5 & 22,3 \\
VBP1100S-800 ${ }^{\circ} \mathrm{C}$ & 331,2 & 1,9 & 22,9 \\
VBP1100S-900 ${ }^{\circ} \mathrm{C}$ & 192,3 & 0,5 & 9,4 \\
\hline
\end{tabular}

scaffolds de VB obtidos pela técnica da espuma, em que o difratograma apresenta picos de cristalinidade com tratamento térmico a $700{ }^{\circ} \mathrm{C}[28]$.

A área superficial (SSA) dos scaffolds foi determinada com o modelo BET, adsorção física das moléculas de gás sobre superfície sólida em multicamadas. A distribuição de diâmetro de poro foi calculada pelo modelo BJH (Barrett, Joyner e Halenda) [29] aplicado para as curvas de dessorção, que confirmou que os scaffods têm estrutura mesoporosa com SSA variando de $73,2 \mathrm{~m}^{2} / \mathrm{g}$ no VBP160S- $800{ }^{\circ} \mathrm{C}$ até $331,2 \mathrm{~m}^{2} / \mathrm{g}$ no VBP1100S- $800{ }^{\circ} \mathrm{C}$. Os scaffolds foram classificados como mesoporosos (Tabela IV), como esperado para os materiais bioativos obtidos pelo método sol-gel, com poros na faixa de 2 a $50 \mathrm{~nm}$, que são bem mais favoráveis do que aqueles completamente densos, porque estes materiais possuem alta área superficial o que resulta em alta bioatividade e elevada tendência à biorreabsorção. As isotermas podem ser identificadas como tipo II, as quais são características de materiais mesoporosos. Mesoporosidade é indicada pela presença de histerese de adsorção/dessorção e pela inclinação da curva de adsorção [30].

\section{Avaliação da citotoxidade dos scaffolds}

A atividade metabólica mitocondrial dos osteoblastos primários foi avaliada pelo ensaio de MTT após $24 \mathrm{~h}$ de exposição dos osteoblastos ao material. Esta análise permite mensurar o metabolismo celular com a adição do MTT. As células viáveis absorvem o tetrazólio de metiltiazol e o metabolizam na mitocôndria utilizando enzimas ativas, que se acumulam sob a forma de cristais de tetrazólio ou formazan, sendo possível diferenciar células viáveis das menos viáveis. Quanto maior a leitura no espectrofotômetro, maior é a taxa metabólica [31]. A Fig. 8 apresenta a viabilidade relativa das células osteoblásticas primárias em contato com os materiais. A viabilidade das células osteoblásticas variou de 96,5\% no VBP1100S-900 ${ }^{\circ} \mathrm{C}$ até $145 \%$ no VBP158S- $900{ }^{\circ} \mathrm{C}$, utilizando como controle negativo células cultivadas no meio sem o material, como $100 \%$ de viabilidade, e como controle positivo células postas no meio de cultura com adição de Triton, substância

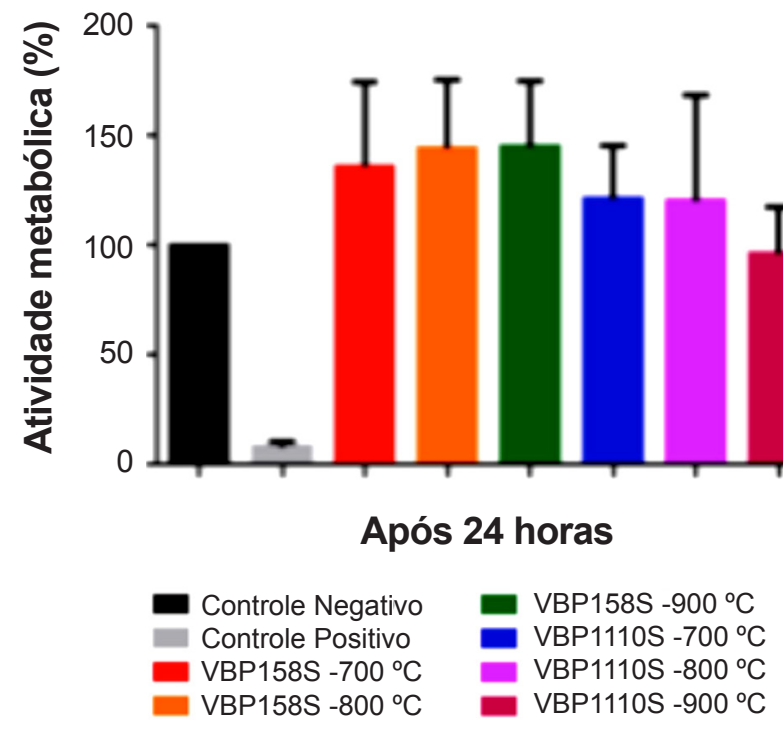

Figura 8: Atividade metabólica dos osteoblastos primários quando expostos aos scaffolds de VB58S e VB100S por $24 \mathrm{~h}$.

[Figure 8: Metabolic activity of the primary osteoblasts when exposed to the VB58S and VB100S scaffolds during 24 h.]

citotóxica. O aumento de temperatura de tratamento nos scaffolds $58 \mathrm{~S}$ não diminuiu a proliferação celular e sim demonstrou um pequeno aumento da atividade metabólica dos osteoblastos. Os scaffolds $100 \mathrm{~S}$ apresentaram atividade celular satisfatória, embora menor que nos scaffolds $58 \mathrm{~S}$; o aumento de temperatura de tratamento ocasionou apenas pequena diminuição da atividade metabólica celular. Com base nesses resultados concluiu-se que os scaffolds $58 \mathrm{~S}$ e 100 S são biocompatíveis, uma vez que a viabilidade mínima aceitável como biocompatível é de 50\% [31]. Os resultados mostraram significado estatístico apenas dos scaffolds e do controle negativo em relação ao controle positivo; não houve diferença estatística significativa entre o controle negativo e os scaffolds, e entre os scaffolds; porém, observa-se que os scaffolds $58 \mathrm{~S}$ e $100 \mathrm{~S}$, e com maior porcentagem o 58S, induziram a proliferação celular das células osteoblásticas primárias, fator de relevância para o sucesso da regeneração óssea e sua utilização na engenharia de tecido ósseo. 


\section{CONCLUSÕES}

A partir dos resultados conclui-se que é possível adicionar agentes porogênicos ao VB obtido via sol-gel e produzir scaffolds para engenharia de tecidos. Os scaffolds obtidos via sol-gel do VB 58 e 100S com adição de parafina 1 são viáveis para regeneração óssea. Apresentaram poros interconectados, com fração de poros abertos superior a 50\% e diâmetro de poros superior a $150 \mu \mathrm{m}$. Os VBP158S-700 ${ }^{\circ} \mathrm{C}$, VBP158S-800 ${ }^{\circ} \mathrm{C}$, VBP158S-900 ${ }^{\circ} \mathrm{C}$, VBP1100S-700 ${ }^{\circ} \mathrm{C}$, VBP1100S-800 ${ }^{\circ} \mathrm{C}$ e VBP1100S- $900{ }^{\circ} \mathrm{C}$ apresentaram diâmetro médio de poros de 269, 290, 278, 442, 400 e $371 \mu \mathrm{m}$, respectivamente, e fração de poros abertos de $56,4 \%, 52 \%, 51 \%, 67,4 \%, 67,6 \%$ e $70 \%$, respectivamente, sendo que a relação porosidade total/poros abertos foi de quase $100 \%$. Os scaffolds apresentaram formato de poros arredondados, abertos em seu interior, o que foi demonstrado através da caracterização tridimensional por MEV e $\mu \mathrm{CT}$. Os scaffolds tiveram estrutura mesoporosa e os mesoporos variaram de $9,4 \mathrm{~nm}$ no VBP1100S-900 ${ }^{\circ} \mathrm{C}$ até $22,9 \mathrm{~nm}$ no VBP1100S- $800{ }^{\circ} \mathrm{C}$. A área superficial variou de $73,2 \mathrm{~m}^{2} / \mathrm{g}$ no VBP158S-800 ${ }^{\circ} \mathrm{C}$ até $331,2 \mathrm{~m}^{2} / \mathrm{g}$ no VBP1100S-800 ${ }^{\circ} \mathrm{C}$. Os scaffolds de VB 58S e $100 \mathrm{~S}$ continuaram amorfos mesmo com o aumento de temperatura de tratamento térmico. Os scaffolds não apresentaram citotoxicidade ao ensaio de MTT e induziram a proliferação das células osteoblásticas primárias, mesmo os scaffolds de VB100S, fatores de relevância para o sucesso da regeneração óssea e sua utilização na engenharia de tecido ósseo.

\section{REFERÊNCIAS}

[1] S.E. Lynch, R.E. Marx, M. Nevins, L.A. Wisner-Lynch, Tissue Engineering Applications in Oral and Maxillofacial Surgery and Periodontics, $2^{\mathrm{a}}$ Ed., Quintessence Publishing, Chicago, EUA (2008) 296.

[2] M.N. Rahaman, D.E. Day, B.S. Bal, Q. Fu, S.B. Jung, L.F. Bonewald, A.P. Tomsia, Acta Biomater. 7, 6 (2011) 2355-2373.

[3] M.M. Pereira, L.L. Hench, Bioactive glass. In: G.E. Wnek, G.L. Bowlin, Eds., Encyclopedia of Biomaterials and Biomedical Engineering, $2^{\text {a }}$ Ed., Marcel Dekker, New York, EUA (2004) 53-62.

[4] A.S. Hoffman, B.D. Ratner, F.J. Schoen, J.E. Lemons, Biomaterials Science, an Introduction to Materials in Medicine, 2 ${ }^{\mathrm{a}}$ Ed., Elsevier, San Diego EUA (2004) 879.

[5] L.L. Hench, N. Roki, M.B. Fenn, J. Mol. Struct. 1073 (2014) 24-30.

[6] L.L. Hench, J. Mater. Sci: Mater. Med. 17 (2006) 967978.

[7] L.C. Gerhardt, A.R. Boccaccini, Mater. 3 (2010) 38673910.
[8] G.L. Jiang, C.R. White, H.Y. Stevens, J.A. Frangos, J. Physiol. Endocrinol. Metab. 283 (2002) 383-389.

[9] D. Bellucci, V. Cannillo, A. Sola, Sci. Sintering 42 (2010) 307-320.

[10] J. Ma, C.Z. Chen, D.G. Wang, X.G. Meng, J.Z. Shj, Ceram. Int. 36, 6 (2010) 1911-1916.

[11] S.C. Owen, M.S. Shoichet, J. Biomed. Mater. Res. 94A, 4 (2010) 1321-1331.

[12] Q. Chen, J. Roether, A. Boccaccini, Tissue engineering scaffolds from bioactive glass and composite materials, In: N. Ashammakhi, R. Reis, F. Chiellini, Eds.; Topics in Tissue Engineering, V. 4 Chap. 6, Expertissues e-books, Oulu, Finland (2008) 1-27.

[13] F. Baino, M. Ferraris, O. Bretcanu, E. Verné, C. VitaleBrovarone, J. Biomater. Appl. 27 (2013) 872-890.

[14] A.R. Studart, U.T. Gonzenbach, E. Tervoort, L.J. Gauckler, J. Am. Ceram. Soc. 89, 6 (2006)1771-1789.

[15] E.P. Santos, C.V. Santilli, S.H. Pulcinelli, J. Non-Cryst. Solids 304, 1-3 (2002) 143-150.

[16] J.R. Jones, Acta Biomater. 9 (2013) 4457-4486.

[17] G. Poologasundarampillai, D. Wang, S. Li, J. Nakamura, R. Bradley, P.D. Lee, M. M. Stevens, D.S. McPhail, T. Kasuga, J.R. Jones, Acta Biomater. 10, 8 (2014) 3733-3746. [18] Y. Kang, S. Kim, A. Khademhosseini, Y. Yang, Biomater. 32, 26 (2011) 6119-6130.

[19] J.L. de M. Machado, L.A. dos Santos, Cerâmica 55, 334 (2009) 216-222.

[20] M.M. Pereira, E.A. Clark, L.L. Hench, J. Mater. Synth. Process. 2, 3 (1994) 189-195.

[21] M.M. Pereira, E.A. Clark, L.L. Hench, J. Biomed. Mater. Res. 28 (1994) 693-698.

[22] J. Ajita, S. Saravanan, N. Selvamurugan, Mater. Sci. Eng. C53 (2015) 142-149.

[23] S. Yang, K. F. Leong, Z. Du, C.K. Chua, Tissue Eng. 7, 6 (2001) 679-689.

[24] F.P.W. Melchels, J. Feijen, D.W. Grijpma, Biomater. 30 (2009) 3801-3809.

[25] A.O. Helminen, H. Korhonen, J.V. Seppala, J. App. Polym. Sci. 86 (2002) 3616-3624.

[26] A. Ronca, "Bioactive Composite Scaffolds for Bone Regeneration: From the Process to the Biological Validation", Tese Dr., Napoli: Università Degli Studi di Napoli "Federico II" (2010) 148p.

[27] H. Yoshikawa, N. Tamai, T. Murase, A. Myoui, J. R. Soc. Interface 6 (2009) 341-348.

[28] M.B. Coelho, M.M. Pereira, J. Biomed. Mater. Res. Part B: Appl. Biomater. 75B, 2 (2005) 451-456.

[29] N.J. Coleman, L.L. Hench, Ceram. Int. 26 (2000) 171178.

[30] K.S.W. Sing, Pure Appl. Chem. 57 (1985) 603-619.

[31] T. Mosmann, J. Immunological Methods 65 (1983) 5563.

(Rec. 22/12/2015, Rev. 19/02/2016, Ac. 09/04/2016) 